\title{
Some Thoughts on an Eddy Current Septum Magnet
}

\author{
Klaus Halbach \\ Argonne National Laboratory and UC Lawrence Berkeley Laboratory
}

\section{Introduction}

It is the purpose of this report to describe the thinking and work that went into thoroughly understanding a specific eddy current septum magnet (and very similar magnets) that will be used in the APS and needs "fixing." The goal is to go beyond mere qualitative understanding and carry the analysis far enough to recognize the real problems of this magnet and to develop the tools to solve them. While it would be very tempting to write this report in very general terms, it would be very difficult (or even impossible) to achieve complete generality, and one would risk losing the focus on this specific magnet that needs to have an improved performance. The discussion contains, however, so many generally useful concepts and procedures that it might further be useful for designers of other eddy current septum magnets both at the APS and elsewhere. Since it is necessary not only to have a deep qualitative understanding of several aspects of this type of magnet, but also to quantitatively assess what has to be done (or should not be done) to achieve the desired performance, fairly heavy use of mathematical tools was made. In doing so it became clear that the extensive use of these tools is essential not only for this purpose, but also to get the required good qualitative understanding of the device. For instance, it turns out that in this particular instance, an important part of the underlying physics (see section 5.2) does not become clear until one seems to run into what one might consider, at first, a mathematical problem, whose resolution is not clear until one has found the solution, which then seems "obvious." Even though iron in the septum has a nonlinear relationship between $B$ and $H$, assuming a constant permeability is sufficient to obtain the information needed to develop a sufficient understanding of the magnet to make some crucial decisions. As will be shown below, the reason is the fact that it is practically impossible to meet the desired performance as soon as the nonlinearity of the iron is sufficiently strong that it has to be taken into account. This means that one has to design the magnet in such a way that saturation during critical times is avoided, and the linear model makes that possible with relatively little pain.

\section{Septum magnet specifications}

The magnet should produce a half sine wave field with a peak amplitude of $7.3 \mathrm{kG}$ and a duration of $T_{s}=330 \mu \mathrm{sec}$ from zero field to zero field in the "inside" region. Up to a short time after the peak excitation, the field in the "outside" region should be less than $12 \mathrm{G}$ or $1 \mathrm{G}$, depending on other operational parameters. The repetition rate is $2 \mathrm{~Hz}$. A very similar magnet, with less demanding outside field level specifications, will be run with a repetition rate of $60 \mathrm{~Hz}$. At the present time, the total septum thickness is $2 \mathrm{~mm}$, and is equally divided between copper $(\mathrm{Cu})$ and iron $(\mathrm{Fe})$. The thickness of the laminations in the yoke is $2 d_{L}=0.36 \mathrm{~mm}$. To achieve the desired performance, all of these parameters can be changed by moderate amounts, except the

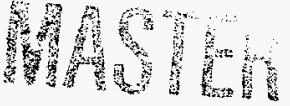




\section{DISCLAIMER}

Portions of this document may be illegible in electronic image products. Images are produced from the best available original document. 
specifications for the peak field inside the magnet and the amplitude of the field leaking through the septum should be considered frozen.

\section{Global considerations}

Figure 1 shows a schematic cross section of the magnet under discussion. One of the often given reasons for preferring to drive a septum in this indirect way over a directly driven septum is the common belief that loss of A-turns in the iron yoke leads to a smaller outside leakage field in the eddy current septum magnet. In order to examine this question, but in particular to obtain a general basic understanding of this type of magnet, we consider a model magnet which, even though different in details, is topologically the same as the magnet under discussion.

Going in Fig. 2 from the iron yoke at the center to the left, it shows the primary coil that is driven by an external power supply, followed by a vacuum region were the field goes from the full value level $H_{1}$ to the reduced (because of A-turn loss in the iron) value $\eta_{1} H_{1}$ next to the inside surface of the septum. Further to the left is the bulk of the septum (which may contain several different materials), followed by the outside septum surface and the outside vacuum region. To the right of the yoke is the same kind of a structure, but with different values for geometrical and related quantities, with the total current in the right side septum conductor being the same (except for sign) as the current in the left side septum conductor.

If one turns on the power supply-driven coil with a current that appears as $\pm_{0}$ in the upper half-magnet shown in Fig. 2, and looks at what happens during very short times compared to the time it takes fields to penetrate deeply into the septa, one finds the following conditions: returning to the left of the center yoke first, and assuming for simplicity finite permeability only in the region where A-turn loss is explicitly indicated, one gets a field $H_{1}$ very close to the coil, with the line integral from the midplane to the pole being exactly $I_{0}$. Because of the finite permeability of the iron in the yoke, the field, and with it its line integral, is smaller by the factor $\eta_{J}<I$ when one is next to the septum. Since eddy currents in the septum will prevent the penetration of the fields into the bulk of the $\mathrm{Cu}$, the total current in that thin skin has to be exactly $\eta_{l} I_{0}$ in order to have no fields inside the $\mathrm{Cu}$. It is useful to remember that this is exactly the same basic process (with different, but conceptually irrelevant, details) that occurs when one has a solid Cu body, like a sphere, and exposes it suddenly to an externally generated field. In Fig. 2 the positive direction for currents is out of the paper, and the positive direction of fields is down, as indicated. On the right side of the yoke the same physics arguments apply, leading to equivalent conditions, but with modified numerical values of all fields and currents, with the exception of $\pm_{0}$. What is particularly interesting is that, in general, $\eta_{2}$ will be different from $\eta_{l}$, thus causing a "problem." Summing the total current out of the paper plane from both parts of the septum conductor considered so far, one necessarily gets $\left(\eta_{2}-\eta_{1}\right) I_{0}$ not equal to zero. Since the currents on the inside boundaries of the septa are given by the fields, which in turn are controlled by the power supply and the iron, and since a current in the bulk of the septa is "illegal" on a very short time scale, one has to have some currents on the outside surfaces of the septa. These currents and the associated fields are also indicated in Fig. 2. The values of $\chi_{1}$ and $\chi_{2}$ are determined by the "total current $=0$ " condition

$$
\eta_{2}-\eta_{1}+\chi_{1}-\chi_{2}=0
$$


and the condition that for very short times the total flux passing through the one turn septum loop has to be zero. Since both the flux through the one turn septum loop as well as the flux through any infinite plane has to be zero, the easiest way to understand and calculate that flux is to look at the total flux outside the septum loop. If (per unit length in the direction perpendicular to the paper) the fields $\chi_{1} H_{1}$ and $\chi_{2} H_{2}$ extend in the outside direction, as far as the associated flux is concerned, by $w_{1}$ and $w_{2}$, the total outside flux would be

$$
F=\chi_{1} H_{1} w_{1}+\chi_{2} H_{2} w_{2}
$$

Assuming no flux in the septum itself for very short times, we can set $F$ equal to zero yielding, together with the total current condition,

$$
\chi_{1}=-\left(\eta_{1}-\eta_{2}\right) /\left(1+H_{1} w_{1} /\left(H_{2} w_{2}\right)\right)
$$

This means that if the loss of A-turns for the two regions is different, there will be a noticeable prompt field outside the septum, except when the denominator is very large.

Even though this model is obviously lacking all the details of the real magnet (which it must in order not to get lost in complicated calculations), it is clear that it correctly describes the basic concepts that underlie the fundamental processes at work. The topology is clearly correct. The actual values of the fields, for instance, are not relevant for understanding how the system works since the total current on a septum surface is independent of the field distribution, but depends only on the line integral along field lines in the vacuum, together with the total current and total flux equal zero conditions. One also has to remember that the events described above occur simultaneously, i.e. we have simply described a self consistent solution that satisfies all the essential electro-magnetic conditions. Left out intentionally are some details that can be important in the real world. The ends are clearly left out in a 2D model and have to be taken into account in the real magnet, but can be easily understood if one follows the concepts from the $2 \mathrm{D}$ model. To be more specific on this particular topic: depending on how the primary coil and the septum conductors are arranged at the ends, one can easily induce flux there directly from one loop into the other, giving an additional term in the flux equation above, and therefore changing the field outside the septum. Conceptually included in the model is, however, the coupling of the eddy currents and field penetration between, in Fig. 2, the septum on the left (1) and the "septum" on the right (2). If, for instance, the field penetrates at a different rate through septum 2 than through septum 1, a current can appear on the left side of septum 1, giving the appearance that the field has penetrated through the septum when, in fact, it has not yet penetrated. That (and similar other things) is the reason why one should not worry if one does not see an undelayed reverse field outside the septum. There would be reason for concern, however, if one makes a change in the magnet that would cause a predictable change in the behavior and that change would not materialize. One can think of a number of such changes, for instance introducing coupling at the ends, or changing $w_{2}$ by introduction of some laminated iron on the side of the real magnet, that is represented by the right side of the model. Since the specific magnet in the APS does not show any behavior that would indicate a prompt field outside the septum, this topic will not be discussed in any more detail. 


\section{Consequences of eddy currents in the iron yoke laminations}

The conclusion of this section will be that eddy currents in the laminations do not present a significant problem for the present set of parameters, but should be re-examined when operating parameters (e.g. the length of the half sine pulse) have to be drastically changed. It is therefore not necessary to derive details in this section, in particular since their substance is implicitly contained in the discussion of the penetration of the field through the septum as well as in Ref. 1.

If one takes $\int \vec{H} \cdot d \vec{s}$ around the dotted path indicated in Fig. $1, \int \vec{H} \bullet d \vec{s}$ from the vacuum field equals the total A-turns enclosed by that loop minus $\int \vec{H} \cdot d \vec{s}$ in the yoke. Since there will be eddy currents in the iron laminations they have to be taken into account if one chooses just any plane for that loop, thus making the calculation of the loss of A-turns in the iron very complicated. To avoid that, one can choose the space between the laminations (which is equivalent to the edge of a lamination) as that plane, giving simply that the loss of A-turns in the laminations is determined by the field at the edge of a lamination. Even though Ref. 1 deals with solid core magnets, the logic and results of that paper are directly applicable to any magnet where the field in the vacuum region is controlled by a coil system and a dominating non-magnetic gap. Assuming a constant permeability $\mu$ in the iron, the relationship between the Laplace (or Fourier) transforms of $B$ at the edge of a lamination and the value $\underline{B}$ of the field averaged over the lamination thickness $2 d_{L}$ is given by

$$
B=B \frac{\varphi}{\tanh (\varphi)}
$$

with

$$
\varphi=\sqrt{p \tau}, \tau=\sigma \mu_{0} \mu d_{L}^{2}
$$

where $p$ is the Laplace variable (or, for a periodic process, $i$ times the circular frequency $\omega$ ), and $\sigma$ the conductivity of the iron. This solution comes from the same differential equations as the ones solved below for the eddy currents in the septum, except the boundary or initial conditions are different. If one assumes for the iron (all in MKS units) $\sigma \mu_{O} \mu=3 \times 10^{4}$ one obtains for $d_{L}=.18 \mathrm{~mm}$ a time constant $\tau=l$ msec. From equ.(1) follows that transients decay with exponentials with time constants $T_{n}=\tau /(n \pi)^{2}$, with $n=1,2, \ldots$.Even the longest of these time constants is sufficiently short that a repetition rate of $60 \mathrm{~Hz}$ cannot drive the laminations into saturation. During the half sine pulse, however, the eddy currents will increase the field at the edge of a lamination significantly over the average value of the field in the lamination. With the same techniques that will be used in section 5 , during a half sine pulse of amplitude one gets (averaged over the thickness of the lamination) the following field at the edge:

$$
\begin{aligned}
& B(t)=B_{0} \sin \left(\omega_{0} t+\alpha\right)+2 \sum_{n=1} \exp \left(s_{n} \omega_{0} t\right) /\left(s_{n}+1 / s_{n}\right) \\
& s_{n}=-(n \pi)^{2} /\left(\omega_{0} \tau\right) .
\end{aligned}
$$


$B_{0}$ and $\alpha$ are, respectively, the amplitude and phase of the edge field for a periodic $\underline{B}$ of amplitude 1 , obtained by evaluating $\varphi / \tanh (\varphi)$ for $\varphi=\left(i \omega_{0} \tau\right)^{1 / 2}$. Figure 3 shows $\underline{B}$ and $B$ during the half sine pulse for $\omega_{0} \tau=10$. It is clear that, as expected from Ref.1, the peak value of $B$ is significantly larger (2.7 times!) than the peak value of $\underline{B}$, and $B$ is proportional to $t^{1 / 2}$ for small $t$. While the large peak value of $B$ cannot be taken very seriously when it becomes large enough to lead to saturation, a large $B$ would indicate that one is in the region of parameter space where one has to be prepared to encounter some difficulties caused by excessive saturation. In order to make a valid assessment of the upper safe limit for $\omega_{b} \tau$, one would have to take the real $B(H)$ of the iron into account, a task that can be undertaken with available numerical analysis tools, but is not particularly pleasant. From a purely academic point of view it is interesting to realize that $B$ reaches a larger value in the reverse direction than the peak value of $\underline{B}$, and this occurs before $\underline{B}$ goes to zero.

\section{Penetration of field through septum}

\subsection{The governing differential equation and its basic solution}

Figure 4 shows schematically a septum consisting of two separate sheets. The orientation and coordinates (but not the subscripts of the fields) match the convention used in Fig. 2. Assuming a structure very long in the $z$-direction, and assuming that the exciting field generated by the (not shown) coil to the right of the septum is uniform, we are dealing with a problem where the quantities of interest depend only on one space coordinate, namely $x$. This assumes, in principle, that the iron gap is also uniform for some distance to the left. In practical terms, that condition is not very important. The magnetic and electric fields $B$ and $E$ are in the $y$ and $z$-directions respectively, and the field is penetrating in the $x$-direction. With $\delta / \delta x$ represented by ', and using again the Laplace variable $p$, Maxwell's equations reduce for this case to:

$$
\begin{array}{ll}
\operatorname{curl}(\vec{H})=\sigma \vec{E} & \Rightarrow \quad H^{\prime}=\sigma E \quad \Rightarrow \quad E=\rho H^{\prime} \\
\operatorname{curl}(\vec{E})=-\vec{B} & \Rightarrow \quad-E^{\prime}=-p \mu_{0} \mu H .
\end{array}
$$

$\sigma$ and $\rho$ are, respectively, conductivity and resistivity of the material, $\mu$ is the permeability (assumed to be constant), and the choice has been made to use $H$ to describe the magnetic field. Equs.(3) can be combined to give

$$
H^{\prime \prime}=k^{2} H \quad ; \quad k^{2}=p \sigma \mu_{0} \mu
$$

The solution is best expressed in terms of hyperbolic sine and cosine functions. In order to simplify the matching of boundary conditions, it is convenient to introduce a status vector $\mathrm{v}$ with two quantities that are continuous as one goes from one material into the other, and the obvious choice for the two components is $H$ and $E=\rho H^{\prime}$. Introducing

$$
\varphi(x)=k x,
$$

the solution to equ.(4) can be written as 


$$
v(x)=\left(\begin{array}{l}
H(x) \\
E(x)
\end{array}\right)=\left(\begin{array}{cc}
C & S /(\rho k) \\
S \rho k & C
\end{array}\right)\left(\begin{array}{l}
H(0) \\
E(0)
\end{array}\right)=M(x) v(0) .
$$

In this and the following equations, $S$ and $C$ denote the hyperbolic sine and cosine functions of $\varphi$. When $x$ in equ.(5.1) is replaced by $d_{1}$ or $d_{2}, \varphi, \tau, S$, and $C$ also carry the appropriate subscript, and $\varphi_{1}$, for instance, becomes

$$
\varphi_{1}=k d_{1}=\sqrt{p \tau_{1}} \quad ; \quad \tau_{1}=\sigma_{1} \mu_{0} \mu_{1} d_{1}^{2} .
$$

Clearly, equs.(5) satisfy equs.(3) and (4), and the general relationship between the status vectors $\mathbf{v}$ at two locations is obtained by multiplying the appropriate matrices to get the matrix $M$ "connecting" the two locations.

For all the problems of interest here, $H(O)$ at point 0 in Fig. 4 can be considered given by the power supply. However, to solve a problem, an additional boundary condition, or equivalently, a relation between boundary conditions, is needed. It is important to fully appreciate the fact that without this additional condition, it is impossible to find a solution to any problem.

\subsection{The second boundary condition}

As the field penetrates through the septum, magnetic field energy appears in the region to the left of point 3 in Fig. 4 . If one expresses that energy $\Gamma$ (per unit length in the $z$-direction) in terms of the field $H_{3}$ at location 3, its value and time derivative are

$$
\Gamma=H_{3}^{2} \mu_{0} a_{\text {eff }} / 2 \quad ; \quad \dot{\Gamma}=\dot{H}_{3} H_{3} \mu_{0} a_{\text {eff }}
$$

This energy transport must equal the power flow expressed by the Poynting vector, i.e.

$$
\dot{H}_{3} H_{3} \mu_{0} a_{\text {eff }}=E_{3} H_{3} \text { gap. }
$$

Using $a_{\text {eff }} / g a p=D$, allocating signs properly, and going to Laplace transform expressions, gives for the general relationship between $E$ and $H$ at the open end of any septum system

$$
E=-p \mu_{0} D H
$$

The meaning of $D$ is clear: if the field at the outer interface of the septum is $H$, the energy stored outside the septum is the same as the field energy associated with the same field in a region of width $D$ in the inside vacuum (or non-magnetic material) region of the septum magnet. Obviously, the value of $D$ depends on the field geometry in the region outside the septum and this aspect of the magnet has to be taken into account when that part of the system is designed. We assume that this has been done and that $D$ is therefore known. Depending on other parameters, $D$ can have a significant effect on the field penetrating the septum, as can be seen from the following qualitative argument: the septum limits the rate with which energy is transmitted through the septum to the region outside the septum. If one increases the volume outside the septum magnet where that energy resides, the field at the septum interface will decrease. 


\subsection{Incorporation of the second boundary condition into the system description}

If the right and left side of the septum are identified by the subscripts 0 and 3 , and if one uses in $v_{3}$ the relation given in equ.(6.3), one gets

$$
\left(\begin{array}{c}
1 \\
-p \mu_{0} D
\end{array}\right) H_{3}=M_{03} v_{0} \quad ; \quad\left(\begin{array}{l}
H_{0} \\
E_{0}
\end{array}\right)=M_{03}^{-1}\left(\begin{array}{c}
1 \\
-p \mu_{0} D
\end{array}\right) H_{3} \text { ， }
$$

giving explicitly $H_{3}$ in terms of the known matrix elements, $D$ and $H_{0}$. In addition one gets the relationship between $E_{0}$ and $H_{0}$ which might be of interest to assess the "load" the septum presents to the exciting coil. If one applies the logic of equ.(7) to the relation between the status vectors at any location within the septum, identified by the subscript 2 , and the open end, one simply replaces the subscript 0 by 2 in equ.(7) and obtains the relationship between $H_{2}$ and $H_{3}$, and also the relationship between $\mathrm{H}_{2}$ and $H_{0}$.

\subsection{Transfer functions for a septum consisting of one non-magnetic $(\mathrm{Cu})$ and one ferromagnetic (Fe) sheet}

Applying equs.(5) and (7) to this septum yields in a straightforward manner the relationship between the field $\mathrm{H}_{0}$ at the inside surface of the septum and the surface at the end (subscript 3) and at any location inside the septum (subscript 2). In this septum, a $\mathrm{Cu}$ (material index 1) sheet is followed by an Fe sheet (material index 2). While equ.(8.1) is very general, we will use for $G$ the appropriate function for the $\mathrm{Cu}-\mathrm{Fe}$ septum, and for $\mathrm{g}$ the specific function that describes the conditions when location 2 is the interface between the non-magnetic $(\mathrm{Cu})$ sheet and the ferromagnetic $(\mathrm{Fe})$ sheet.

$$
\begin{aligned}
& H_{3}=H_{0} / G \quad ; \quad H_{2}=H_{3} g=H_{0} g / G, \text { with } \\
& G=C_{2} C_{1}+S_{2} S_{1} b_{2}+\psi\left(C_{2} S_{1} b_{2}+S_{2} C_{1}\right), \\
& g=C_{2}+\psi S_{2} .
\end{aligned}
$$

In these equations, $C_{2}, S_{2}$ and $C_{1}, S_{1}$ are the hyperbolic cosine and sine functions for the arguments $\varphi_{2}$ and $\varphi_{1}$, with

$$
\varphi_{n}=\sqrt{p \tau_{n}}, \tau_{n}=\sigma_{n} \mu_{0} \mu_{n} d_{n}^{2} \quad, \quad b_{1}=\varphi_{2} / \varphi_{1}=\sqrt{\rho_{1} \sigma_{2} \mu_{2}} d_{2} / d_{1}
$$

In addition we use

$$
\psi=a_{1} \varphi_{1} / b_{2} \quad, \quad a_{1}=D / d_{1} \quad, \quad b_{2}=\sqrt{\rho_{2} \mu_{2} / \rho_{1}}
$$

\subsection{Reference numbers}

To make it easy to do numerical calculations quickly, we use the following (MKS) numbers, with subscripts 1 and 2 referring to $\mathrm{Cu}$ and $\mathrm{Fe}$ :

$$
\sigma_{1} \mu_{0}=64, \sigma_{2} \mu_{0}=6.4, \mu_{2}=1000
$$


This leads to

$$
b_{1}=\varphi_{2} / \varphi_{1}=10 d_{2} / d_{1}, b_{2}=100
$$

This gives for $d_{2}=d_{1}=1.25 \mathrm{~mm}$ (which we will use most of the time) time constants $\tau_{1}=1 \times 10^{-4}$ and $\tau_{2}=1 \times 10^{-2}$ seconds, and $\omega_{0} \tau_{1}=1, \omega_{0} \tau_{2}=100$. In addition, we will generally use $a_{l}=10$.

\subsection{Some properties of a pure Cu septum}

Setting $d_{2}=0$ in equs.(8) for a pure Cu septum, or applying equs.(5.2) and (7) directly to a one component structure, yields

$$
G=C_{1}+a_{1} \varphi_{1} S_{1}
$$

The Laplace transform for a single half sine pulse of amplitude one and duration $T_{0}$ starting at $t=0$ with $\omega_{0}=\pi / T_{0}$ is:

$$
H_{0}=\omega_{0}\left(1+\exp \left(-p T_{0}\right) /\left(p^{2}+\omega_{0}^{2}\right)\right.
$$

One method to obtain the field at the end of the septum consists of finding all singularities of $G(p)$ and summing up all their contributions to the inverse Laplace transform integral. These singularities are obviously at $\pm i \omega_{0}$ and where $G(p)=0$. One can show (with some work) that all of these roots are on the negative real axis in the complex p-plane. They are easily obtained by setting in equ. (10.1)

$$
\varphi_{1}=\sqrt{p \tau_{1}}=i \alpha
$$

and solving

$$
\tan \alpha=1 /\left(a_{1} \alpha\right)
$$

Since it is very likely that $a_{1} \geq 10$, it is clear when one imagines plotting both the left and right side of equ.(10.4) that the solutions are in very good approximation given by

$$
\alpha_{0}=1 / \sqrt{a_{1}}
$$

giving

(10.6) $-p_{0}=1 / t_{0}=1 /\left(\tau_{1} a_{1}\right)$ and

(10.7) $-p_{\mathrm{n}}=1 / t_{\mathrm{n}}=(n \pi)^{2} / \tau_{1}$

for the remaining time constants for $n>0$.

The solution for $H_{3}$ in the time domain for half sine pulse excitation (i.e. when $H_{0}$ is given by equ.(10.2)) becomes 


$$
\begin{aligned}
& 0<t<T_{0}: H_{3}=B_{0} \sin \left(\omega_{0} t+\beta\right)+2 \sum_{n=0} \frac{\exp \left(-t / t_{n}\right)}{\left(p_{n} / \omega_{0}+\omega_{0} / p_{n}\right) G_{n}^{\prime} p_{n}} \\
& T_{0}<t: H_{3}=2 \sum_{n=0} \frac{\exp \left(-t / t_{n}\right)\left(1+\exp \left(T_{0} / t_{n}\right)\right)}{\left(p_{n} / \omega_{0}+\omega_{0} / p_{n}\right) G_{n}^{\prime} p_{n}} .
\end{aligned}
$$

In these expressions, $B_{0}$ and $\beta$ are defined by

$$
1 / G\left(i \omega_{0}\right)=B_{0} \exp (i \beta)
$$

and $G_{n}^{\prime}$ by

$$
G_{n}^{\prime}=(d(G(p)) / d p)_{p=p_{n}}
$$

\subsection{Discussion of $\mathrm{Cu}$ septum properties}

One of the most noteworthy properties of the $\mathrm{Cu}$ septum is the time constant $t_{0}$ and its relation to $D$ (through $a_{1}=D / d_{1}$ ) and to $t_{1}$ : for a value of 10 for $a_{1}, t_{0}$ is about 100 times larger than $t_{l}$, the time constant that one expects to control the longest decay time of the eddy currents in the septum (if one used somewhat simplistic logic). This indicates that the design of the system beyond the end of the septum can have a noticeable effect on the dynamics of a pure $\mathrm{Cu}$ septum. Unfortunately it is, in general, not easy to obtain from equs.(11) information with good accuracy for $t / \tau_{l}<<l$ since one has to take a large number of terms in equ.(11.1) into account. In the case of the pure $\mathrm{Cu}$ septum magnet, that difficulty is ameliorated by the fact that it easy to obtain the roots of $G=0$. This is not the case for the Cu-Fe composite septum, and in Section 5.8.3 a better method to deal with that difficulty will be developed. Figure 5 shows the field at the outside face of the $\mathrm{Cu}$ septum during the half sine pulse for $\omega_{0} \tau_{l}=4$ (corresponding to a thickness of $2.5 \mathrm{~mm}$ for our reference numbers) and $a_{l}=10$. It is obvious that the performance of this pure $\mathrm{Cu}$ septum falls short of the required performance by about a factor of 10 . Evaluation of the field for a wide range of parameters shows that $H_{3}$ at $\omega_{0} t=\pi / 2$ is, as expected, roughly inversely proportional to $a_{i}$.

\subsection{The compound Cu-Fe septum}

\subsubsection{Introduction}

It is clear from the previous section that for the magnet under discussion it is necessary to back up the $\mathrm{Cu}$ septum, toward the outside region, with an iron septum. The iron obviously cannot provide significant benefits if it is badly saturated during critical times. There are essentially two reasons why the iron septum can be driven into saturation: If, before reaching the peak excitation, the iron at the $\mathrm{Cu}-\mathrm{Fe}$ interface reaches a field $\mathrm{H}_{2}$ that is larger than the value where saturation begins (typically of order $\mu_{0} H_{2}=10 \mathrm{G}$ ), the Fe septum will saturate and the stray field in the outside region will increase dramatically. Even if that does not occur, but the next pulse appears before the eddy currents in the structure have decayed to a very small value, the fields in the iron can cumulatively increase until saturation limits that process, meaning that the permeability will drastically go down, and again the stray field to the outside will increase. 


\subsubsection{Time constants of the $\mathrm{Cu}-\mathrm{Fe}$ septum}

Finding the roots of $G=0$ is considerably more difficult for equ.(8.2) when the simplifications that gave equ.(10.1) are not made. But again, there are only solutions that are on the negative real axis of the complex $p$ plane. To find them we introduce analogously to equ.(10.3)

$$
\varphi_{1}=i \alpha_{1}, \quad \varphi_{2}=i \alpha_{2}
$$

with $\alpha_{2} / \alpha_{1}=b_{1}$. To find the smallest root (giving the longest decay time), we assume that $\alpha_{2}<<1$ and find from equ.(8.2) in very good approximation

$$
\begin{aligned}
& \alpha_{2}^{2}=b_{1} / b_{2}, p_{0}=-1 /\left(\sqrt{\tau_{1} \tau_{2} b_{2}}\right) \\
& -p_{0}=1 / t_{0}=I /\left(\sigma_{1} \mu_{0} \mu_{2} d_{1} d_{2}\right)
\end{aligned}
$$

It should be noted that, in this approximation, the (longest) time constant is independent of $\sigma_{2}$. Although for non-zero conductivity $\sigma_{2}$ this time constant is weakly dependent on $\sigma_{2}$, the answer for $p_{0}$ is the same when one sets this conductivity exactly to zero. When doing so it becomes clear that equ.(12.3) is very closely related to equ.(10.6) and the second boundary condition introduced in Section 5.2: letting $\sigma_{2}$ go to zero in equ.(8.2) leads to the same equation as equ.(10.1), except $a_{1}$ has been replaced by $\left(D+\mu_{2} d_{2}\right) / d_{1}$, expressing the increased stored energy in the non-conducting medium beyond the end of the $\mathrm{Cu}$ septum. The information obtained for the pure $\mathrm{Cu}$ septum therefore also applies to a compound $\mathrm{Cu}-\mathrm{Fe}$ septum if the iron has zero conductivity, and if the appropriate value for $a_{1}$ is used. The second noteworthy fact is the very large value of $t_{0}(0.1 \mathrm{sec})$ for the reference data set. The danger of saturating the iron in the septum because of the overlapping effects of successive pulses is a subject of grave concern for repetition frequencies of the order of more than a few pulses per second, and has to be taken very seriously. One of the possible remedies is a properly designed reset pulse of reverse polarity. One of the reset pulse design options that one should consider is to leave the septum iron at the beginning of the "forward" pulse close to saturation in the reverse direction, giving the iron in the septum a larger dynamical range. It would therefore be advisable to be able to change timing and amplitude of that reset pulse experimentally, since this analysis gives a very good understanding of the processes at work and the rough numbers. But one also has to remember that they are not more than just rough numbers.

In order to see where the other roots are, it is useful to re-write equ.(8.2), with the lower case $c$ and $s$ now describing the trigonometric functions of $\alpha$ and $\gamma=\alpha_{2} a_{l} /\left(b_{1} b_{2}\right)$ :

$$
G / b_{2}=c_{1}\left(c_{2}-\gamma s_{2}\right) / b_{2}-s_{1}\left(s_{2}+\gamma c_{2}\right)
$$

Because $b_{2}$ is so large, $G=0$ is essentially satisfied when the second parenthesis is zero, unless $s_{1}$ is very small. This means that the first "few" (i.e. $b_{l}$ ) solutions are obtained in reasonably good approximation from $s_{2}+\gamma c_{2}=0$, i.e.

$$
\alpha_{n}=n \pi, n>0
$$

giving 


$$
-p_{\mathrm{n}}=1 / t_{\mathrm{n}}=(n \pi)^{2} / \tau_{2} \quad, \quad n>0
$$

It should be noticed that these roots are, for all intents and purposes, independent of the properties of the $\mathrm{Cu}$. Again, as in the pure $\mathrm{Cu}$ septum case, the ratio $t_{0} / t_{l}=\pi^{2} b_{2} / b_{1}$ is of order 100 . Figure 6 shows $G(\alpha)$ for the reference numbers $a_{1}=10, b_{1}=10, b_{2}=100$. The root at $\alpha_{0}$ does not show up on that plot. The two closely spaced roots at $\alpha \approx 10 \pi$ are obviously caused by the Cu properties.

\subsubsection{Short time fields}

While the calculation of the fields for the compound septum with expressions similar to equs.(11) is a good procedure to obtain the long time behavior of the fields of the Cu-Fe septum magnet, it is not a good method to evaluate the fields during the half sine pulse, i.e. during short times. There are three main difficulties. The first is the fact that because the fields are very small for short times at the outside septum surface, one has to take a very large number of exponentially decaying terms into account since for small $t$ one has to go to very large solutions of the characteristic equation in order to get very small contributions from these exponentials. As a consequence, one gets very poor accuracy, and sometimes even nonsensical data because of roundoff errors. The second problem is a direct consequence of the first: when one has that kind of a problem, one generally learns very little from the formulae. The third problem is more a difficulty related to convenience: it becomes difficult to find the very large number of solutions to a characteristic equation that is much more complicated than the characteristic equation for the pure $\mathrm{Cu}$ septum, especially if one has to do so for a wide range of septum parameters. For these reasons, a different approach is used to perform the inverse Laplace transforms necessary to obtain information about the fields during the pulse.

The transfer functions, given by equs.(8), can be expanded for values of complex $p$ with large positive real values. Since the exponentials dominate the expressions, one needs only to replace the hyperbolic functions by 0.5 times the appropriate exponential (and prove later that that assumption is justified). That gives in this case in very good approximation for the $\mathrm{Cu}-\mathrm{Fe}$ interface $\left(\mathrm{H}_{2} / \mathrm{H}_{0}\right)$ and the end of the septum $\left(\mathrm{H}_{3} / \mathrm{H}_{0}\right)$

$$
\begin{aligned}
& H_{2} / H_{0}=2 \exp \left(-\varphi_{1}\right) / b_{2} \\
& H_{3} / H_{0}=\frac{4}{b_{2}} \frac{\exp \left(-\varphi_{1}-\varphi_{2}\right)}{1+\psi}
\end{aligned}
$$

The response to a delta function pulse, giving good insight into the properties of the septum, is given by inserting the appropriate values given by equs.(13) into the right hand side of equs.(A1) and (A3) in the Appendix. It is interesting to note that the field at the $\mathrm{Cu}-\mathrm{Fe}$ interface in response to a delta function pulse has a shape (equ.(A1)) that is not only very simple (and "violent," considering how $t$ appears in the exponent!) but, for all intents and purposes, independent of the septum parameters since they only affect the amplitude and the time scale. The peak of that response function occurs at 1/6 times the time constant that appears with $p$ in the exponent of equ.(13.1). The response to a delta function at the end of the septum has, through $\psi$, an extra degree of freedom. By examining equs. (A1) and (A3) it is clear that as $a$ in equ. (A3) changes from very large values to small values, the character of the response changes from the behavior of the right side of equ. (A1) to that of the right side of equ. (A3) for the case $a=0$, when 
the maximum occurs at $1 / 2$ times the time constant in the exponent. Since the quantity $a$ in equ. (A3) corresponds to $b_{2}\left(1+b_{1}\right) / a_{1}(\gg 1)$ for the end of the compound septum (not the pure $\mathrm{Cu}$ septum, where $a=1 / a_{1}<1$ !), the response shape there will not be very different from the shape of the response for the $\mathrm{Cu}-\mathrm{Fe}$ interface. The time constant for the end will, however, be $\left(1+b_{1}\right)^{2}$ times larger for the end than for the $\mathrm{Cu}-\mathrm{Fe}$ interface, because of the exponent in equ.(13.2). While this very long time cannot be taken seriously because of the imperfections of this model, the times to the maximum value of the delta function response give some indication of the behavioral trends of the responses to delta function excitation. One must also remember that while the time to the peak of the response at the $\mathrm{Cu}-\mathrm{Fe}$ interface is a very well known time, depending only on conductivity and thickness of the $\mathrm{Cu}$ septum, the time to the peak response at the end of the septum is a very "soft" number since it depends strongly on the permeability of the iron. Figure 7 shows the response at the $\mathrm{Cu}-\mathrm{Fe}$ interface to delta function excitation, with Fig. 8 showing the very early part of that function. This makes it clear that very early virtually nothing penetrates. This in turn explains the great difficulty in accurately describing that behavior with the type of expansion used in equ.(11).

To have mathematically easily managed expressions for the inverse transforms that adequately represent the half sine pulse for the duration of that pulse,

$$
H_{0}=-\sum_{n=1}^{3}\left(-\frac{\omega_{0}^{2}}{p^{2}}\right)^{n}
$$

has been chosen, corresponding in the time domain to the first three terms in the Taylor series expansion of the sine function. The inverse transforms are then readily executed with the prescriptions given in the Appendix. Figures 9 and 10 show the field at the $\mathrm{Cu}-\mathrm{Fe}$ interface and the end of the septum during the half sine excitation for the optimized parameters worked out in the following section. These figures, together with the previous figures explain again why it is so hard to calculate these very early fields accurately with the methods explored first.

It is interesting that even though the fields in the iron laminations and the fields in the septum are governed by the same differential equation, the short time behavior is vastly different for the two kinds of systems: the initial time derivatives are infinite to all orders in one case, and zero to all orders in the other case. The difference, of course, lies in the initial or boundary conditions.

\section{Improved design parameters}

From the content of the previous sections, it is clear that in order to reduce the stray field at the end of the septum to tolerable levels at the peak of the pulse, one has to use iron with large permeability, i.e. iron that is not saturated. The latter requirement means that the field at the interface should not be very much larger than 0.001 to 0.002 times the primary peak field amplitude. The following table lists, at the time of the peak of the half sine pulse, the interface field and end field for a peak pulse field normalized to one for a total septum thickness of 2,3, and 3.5 $\mathrm{mm}$, and for varying fractions of the total thickness occupied by $\mathrm{Cu}$, with the remainder being iron. The fields behave as expected, with the caution to take not with a grain but a bag of salt the extremely small end fields for some of the parameter combinations. This table makes it clear that it is not possible to achieve the needed low field at the interface for a total septum thickness of $2 \mathrm{~mm}$, 
but that one should consider going at least to a total septum thickness of $3 \mathrm{~mm}$, with $90 \%$ of the total being $\mathrm{Cu}$. The choice does not have to be satisfied exactly since one could trade off pulse length against septum thickness, in the extreme case leaving the total septum thickness as it is, increasing the $\mathrm{Cu}$ share to $90 \%$, and reducing the pulse length to $4 / 9$ of its present value. To actually arrive at a proper decision about the septum thickness, other engineering judgments (like strength of materials, eddy currents in the yoke laminations, etc.) should be taken into consideration as well.

$\begin{array}{llll}\text { Thickness } & \text { Fract } & \text { Interface } & \text { End } \\ 2 & 0.5 & 1.09 \mathrm{E}-2 & 2.93 \mathrm{E}-09 \\ 2 & 0.7 & 8.09 \mathrm{E}-3 & 6.62 \mathrm{E}-06 \\ 2 & 0.9 & 5.82 \mathrm{E}-3 & 1.49 \mathrm{E}-03 \\ 3 & 0.5 & 7.47 \mathrm{E}-3 & 1.98 \mathrm{E}-16 \\ 3 & 0.7 & 4.47 \mathrm{E}-3 & 2.28 \mathrm{E}-09 \\ 3 & 0.9 & 2.51 \mathrm{E}-3 & 1.13 \mathrm{E}-04 \\ 3 & 0.95 & 2.15 \mathrm{E}-3 & 7.53 \mathrm{E}-04 \\ 3.5 & 0.7 & 3.22 \mathrm{E}-3 & 1.63 \mathrm{E}-11 \\ 3.5 & 0.9 & 1.57 \mathrm{E}-3 & 2.47 \mathrm{E}-05 \\ 3.5 & 0.95 & 1.29 \mathrm{E}-3 & 2.83 \mathrm{E}-04\end{array}$

\section{Summary, comments, and recommendations}

- Even though the specific magnet of concern does not seem to have any problems at the ends, one has to be sure to not introduce new difficulties when modifying the magnet. (Sect.3)

- Even though eddy currents in the laminations are unlikely to cause any problems now, the magnet is probably not very far from suffering detrimental consequences from eddy currents in the laminations. A significant decrease of the pulse width may, for instance, cause difficulties that have to be examined in more detail (non-linear $B(H)$ !) than was done in Sect.4

- Although the design of the region outside the septum is not of great importance for a septum magnet that uses iron in the septum, the outside geometry is very important for a septum that does not contain iron. (Sect.5.2)

- The long time behavior of septum magnets with and without iron is controlled by a very long time constant that is typically 100 times longer than the next smaller time constant. In the case of a $\mathrm{Cu}-\mathrm{Fe}$ compound septum, this very long time constant can cause saturation of the iron unless a properly designed reset pulse is used. All indications are that this reset pulse is vital.(Sect.5.8). A separate note describes some simple practical design considerations for the reset pulse.

- Since, at the time of the peak of the half sine pulse, the field at the $\mathrm{Cu}-\mathrm{Fe}$ interface should not exceed the value that would lead to saturation of the iron, it is very important to design carefully the total septum thickness, the apportioning of that thickness to $\mathrm{Cu}$ and $\mathrm{Fe}$, and the pulse length. This design should not be done in isolation, but in concert with other engineering considerations. (Sect.6) 


\section{Acknowledgments}

This report is a consequence of a technical meeting at the APS on 2/3/94 during which I benefited greatly from interactions with virtually all participants, but in particular from discussions with Erwin Rodger (BNL) and Larry Turner (ANL/APS). At LBL I profited from discussions with some of the ALS staff, and especially from Brian M. Kincaid and Ross Schlueter who are always willing to listen, discuss with me the fine points, and generally give me generous support.

\section{Appendix A: Some Laplace transforms that are important for short time expansions}

The listed inverse Laplace transforms and related definitions are, with some modifications, extracted from the Handbook of Mathematical Functions, (M. Abramowitz, I. A. Stegun), with notation that is also slightly different from the notation used in the Handbook. In the following, to the right of $\Rightarrow$ is printed the inverse Laplace transform of the expression printed to the left of $\Rightarrow$.

$$
\begin{aligned}
& \exp (-k \sqrt{p}) \Rightarrow \frac{k}{2 \sqrt{\pi t^{3}}} \exp \left(-u^{2}\right) \quad, \quad u=\frac{k}{2 \sqrt{t}} \quad, \quad k>0 \\
& \frac{\exp (-k \sqrt{p})}{p^{n}} \Rightarrow(4 t)^{n-1} \operatorname{erfc}_{2(n-1)}(u) \quad, \quad n=m / 2, m=1,2 \cdots \\
& \frac{\exp (-k \sqrt{p})}{a+\sqrt{p}} \Rightarrow \exp \left(-u^{2}\right)\left(\frac{1}{\sqrt{\pi t}}-a \operatorname{erfcs}_{o}(a \sqrt{t}+u)\right)
\end{aligned}
$$

In these expressions, the following abbreviations and definitions have been used:

$$
\operatorname{erfc}_{n}(u)=\int_{u}^{\infty} \operatorname{erfc}_{n-1}(x) d x \quad, \quad \operatorname{erfc}_{-1}(u)=\frac{2}{\sqrt{\pi}} \exp \left(-u^{2}\right)
$$

This means that $\operatorname{erfc}_{0}(u)$ is the "straight" complementary error function erfc(u). For the computer evaluations done for this report, $\operatorname{erfc}_{\mathrm{n}}(u)$ is evaluated by using the (backward) recursion

$$
\operatorname{erfc}_{n-2}(u)=2\left(n \operatorname{erfc}_{n}(u)+u \operatorname{erfc}_{n-1}(u)\right)
$$

To appreciate somewhat the behavior of these functions, it is instructive to see the asymptotic expansion of the erfc $\mathrm{n}_{\mathrm{n}}$ functions for large argument:

$$
\operatorname{erfc}_{n}(u)=\frac{2}{\sqrt{\pi}} \frac{\exp \left(-u^{2}\right)}{(2 u)^{n+1}} \sum_{m=0}^{\infty} \frac{(-1)^{m}(2 m+n) !}{n ! m !(2 u)^{2 m}}
$$


It also turns out to be very convenient, when writing the equations as well as programming them, to introduce the function

$$
\operatorname{erfcs}_{n}(u)=\exp \left(u^{2}\right) \operatorname{erfc}_{n}(u)
$$

If one has to do the inverse transform of the expression on the left side of equ.(A3) multiplied by a polynomial in $1 / p$, a partial fraction expansion leads to expressions that can be transformed by applying equs.(A2) and (A3). This is, however, only necessary when $a$ in equ.(A3) is neither very large nor very small. When $a$ is very large, $a+V_{p}$ can be replaced by $a$, and when $a$ is very small, $a+V_{p}$ can be replaced by $V_{p}$, making the (not really difficult, but distinctly unpleasant) partial fraction expansion unnecessary in both cases.

\section{References}

1) K. Halbach, NIM 107 (1973) 529

Comments to printed text on all graphical representations of functions:

The first line gives date and time of computer run, and name of program.

The second line gives abscissa and ordinate of the lower left and upper right comers of the graph.

The rest is graph-specific information, such as parameter values.

\section{Appendix B: Section Titles and List of Numbered Equations}

\section{Section titles}

1) Introduction

2) Septum magnet specifications

3) Global considerations.

4) Consequences of eddy currents in the iron yoke laminations.

5) Penetration of field through septum

5.1) The governing differential equation and its basic solution

5.2) The second boundary condition

5.3) Incorporation of the second boundary condition into the system description

5.4) Transfer functions in Laplace transform domain for a septum consisting of one non-magnetic

(Cu) and one ferromagnetic ( $\mathrm{Fe}$ ) sheet

5.5) Reference numbers

5.6) Some properties of a pure $\mathrm{Cu}$ septum.

5.7) Discussion of $\mathrm{Cu}$ septum properties

5.8) The compound $\mathrm{Cu}-\mathrm{Fe}$ septum

5.8.1) Introduction

5.8.2) Time constants of the $\mathrm{Cu}-\mathrm{Fe}$ septum

5.8.3) Short time fields

6) Improved design parameters

7) Summary, comments, and recommendations

Appendix A: Some Laplace transforms that are important for short time expansions Appendix B: Section Titles and List of Numbered Equations 


\section{List of all numbered equations}

(1.2) $\varphi=\sqrt{p \tau}, \tau=\sigma \mu_{0} \mu d_{L}^{2}$,

(2.1) $B(t)=B_{0} \sin \left(\omega_{0} t+\alpha\right)+2 \sum_{n=1} \exp \left(s_{n} \omega_{0} t\right) /\left(s_{n}+1 / s_{n}\right)$

(2.2) $s_{n}=-(n \pi)^{2} /\left(\omega_{0} \tau\right)$

(3.1) $\operatorname{curl}(\vec{H})=\sigma \vec{E} \quad \Rightarrow \quad H^{\prime}=\sigma E \quad \Rightarrow \quad E=\rho H^{\prime}$

(3.2) $\operatorname{curl}(\vec{E})=-\vec{B} \quad \Rightarrow \quad-E^{\prime}=-p \mu_{0} \mu H$

(4) $H^{\prime \prime}=k^{2} H \quad ; \quad k^{2}=p \sigma \mu_{0} \mu$

(5.1) $\varphi(x)=k x$,

(6.2) $\quad \dot{H}_{3} H_{3} \mu_{0} a_{\text {eff }}=E_{3} H_{3}$ gap.

(6.3) $E=-p \mu_{0} D H$.

(7) $\left(\begin{array}{c}1 \\ -p \mu_{0} D\end{array}\right) H_{3}=M_{03} \nu_{0} \quad ; \quad\left(\begin{array}{c}H_{0} \\ E_{0}\end{array}\right)=M_{03}^{-1}\left(\begin{array}{c}1 \\ -p \mu_{0} D\end{array}\right) H_{3}$ ，

(8.1) $H_{3}=H_{0} / G \quad ; \quad H_{2}=H_{3} g=H_{0} g / G$

(8.2) $G=C_{2} C_{1}+S_{2} S_{1} b_{2}+\psi\left(C_{2} S_{1} b_{2}+S_{2} C_{1}\right)$

(8.3) $g=C_{2}+\psi S_{2}$.

(8.4) $\varphi_{n}=\sqrt{p \tau_{n}}, \tau_{n}=\sigma_{n} \mu_{0} \mu_{n} d_{n}^{2} \quad, b_{1}=\varphi_{2} / \varphi_{1}=\sqrt{\rho_{1} \sigma_{2} \mu_{2}} d_{2} / d_{1}$

(8.5) $\psi=a_{1} \varphi_{1} / b_{2}, a_{1}=D / d_{1}, b_{2}=\sqrt{\rho_{2} \mu_{2} / \rho_{1}}$

(9.1) $\sigma_{1} \mu_{0}=64, \sigma_{2} \mu_{0}=6.4, \mu_{2}=1000$

(9.2) $b_{1}=\varphi_{2} / \varphi_{1}=10 d_{2} / d_{1}, b_{2}=100$.

(10.1) $G=C_{1}+a_{1} \varphi_{1} S_{1}$.

(10.2) $H_{0}=\omega_{0}\left(1+\exp \left(-p T_{0}\right) /\left(p^{2}+\omega_{0}^{2}\right)\right.$

(10.3) $\varphi_{1}=\sqrt{p \tau_{1}}=i \alpha$

(10.4) $\tan \alpha=I /\left(a_{1} \alpha\right)$

(10.5) $\alpha_{0}=1 / \sqrt{a_{1}}$

(10.6) $-p_{0}=I / t_{0}=I /\left(\tau_{1} a_{1}\right)$

(10.7) $-p_{\mathrm{n}}=1 / t_{\mathrm{n}}=(n \pi)^{2} / \tau_{1}$

(11.1) $0<t<T_{0}: H_{3}=B_{0} \sin \left(\omega_{0} t+\beta\right)+2 \sum_{n=0} \frac{\exp \left(-t / t_{n}\right)}{\left(p_{n} / \omega_{0}+\omega_{0} / p_{n}\right) G_{n}^{\prime} p_{n}}$ 
(11.2) $T_{0}<t: H_{3}=2 \sum_{n=0} \frac{\exp \left(-t / t_{n}\right)\left(1+\exp \left(T_{0} / t_{n}\right)\right)}{\left(p_{n} / \omega_{0}+\omega_{0} / p_{n}\right) G_{n}^{\prime} p_{n}}$.

(11.3) $1 / G\left(i \omega_{0}\right)=B_{0} \exp (i \beta)$

(11.4) $G_{n}^{\cdot}=(d(G(p)) / d p)_{p=p_{n}}$.

(12.1) $\varphi_{1}=i \alpha_{1}, \varphi_{2}=i \alpha_{2}$

(12.2) $\alpha_{2}^{2}=b_{1} / b_{2}, p_{0}=-1 /\left(\sqrt{\tau_{1} \tau_{2} b_{2}}\right)$

(12.3) $\quad-p_{0}=1 / t_{0}=1 /\left(\sigma_{1} \mu_{0} \mu_{2} d_{1} d_{2}\right)$.

(12.4) $G / b_{2}=c_{1}\left(c_{2}-\gamma s_{2}\right) / b_{2}-s_{1}\left(s_{2}+\gamma c_{2}\right)$

(12.5) $\alpha_{\mathrm{n}}=n \pi, n>0$

(12.6) $-p_{\mathrm{n}}=1 / t_{\mathrm{n}}=(n \pi)^{2} / \tau_{2}, n>0$

(13.1) $\quad H_{2} / H_{0}=2 \exp \left(-\varphi_{1}\right) / b_{2}$

$H_{3} / H_{0}=\frac{4}{b_{2}} \frac{\exp \left(-\varphi_{1}-\varphi_{2}\right)}{1+\psi}$.

(13.4)

$$
H_{0}=-\sum_{n=1}^{3}\left(-\frac{\omega_{0}^{2}}{p^{2}}\right)^{n}
$$

(A1)

$$
\exp (-k \sqrt{p}) \Rightarrow \frac{k}{2 \sqrt{\pi t^{3}}} \exp \left(-u^{2}\right) \quad, \quad u=\frac{k}{2 \sqrt{t}} \quad, k>0
$$

(A2) $\frac{\exp (-k \sqrt{p})}{p^{n}} \Rightarrow(4 t)^{n-1} \operatorname{erfc}_{2(n-1)}(u) \quad, \quad n=m / 2, m=1,2 \cdots$

$$
\frac{\exp (-k \sqrt{p})}{a+\sqrt{p}} \Rightarrow \exp \left(-u^{2}\right)\left(\frac{1}{\sqrt{\pi t}}-a \operatorname{erfs}_{o}(a \sqrt{t}+u)\right)
$$

(A4) $\quad \operatorname{erfc}_{n}(u)=\int_{u}^{\infty} \operatorname{erfc}_{n-1}(x) d x \quad, \quad \operatorname{erfc}_{-1}(u)=\frac{2}{\sqrt{\pi}} \exp \left(-u^{2}\right)$.

(A5) $\operatorname{erfc}_{n-2}(u)=2\left(n \operatorname{erfc}_{n}(u)+u \operatorname{erfc}_{n-1}(u)\right)$.

(A6)

$$
\operatorname{erfc}_{n}(u)=\frac{2}{\sqrt{\pi}} \frac{\exp \left(-u^{2}\right)}{(2 u)^{n+1}} \sum_{m=0}^{\infty} \frac{(-1)^{m}(2 m+n) !}{n ! m !(2 u)^{2 m}}
$$

(A7) $\operatorname{erfcs}_{n}(u)=\exp \left(u^{2}\right) \operatorname{erfc}_{n}(u)$.

$\begin{array}{llll}\text { Thickness } & \text { Fract } & \text { Interface } & \text { End } \\ 2 & 0.5 & 1.09 \mathrm{E}-2 & 2.93 \mathrm{E}-09 \\ 2 & 0.7 & 8.09 \mathrm{E}-3 & 6.62 \mathrm{E}-06 \\ 2 & 0.9 & 5.82 \mathrm{E}-3 & 1.49 \mathrm{E}-03 \\ 3 & 0.5 & 7.47 \mathrm{E}-3 & 1.98 \mathrm{E}-16 \\ 3 & 0.7 & 4.47 \mathrm{E}-3 & 2.28 \mathrm{E}-09 \\ 3 & 0.9 & 2.51 \mathrm{E}-3 & 1.13 \mathrm{E}-04 \\ 3 & 0.95 & 2.15 \mathrm{E}-3 & 7.53 \mathrm{E}-04 \\ 3.5 & 0.7 & 3.22 \mathrm{E}-3 & 1.63 \mathrm{E}-11 \\ 3.5 & 0.9 & 1.57 \mathrm{E}-3 & 2.47 \mathrm{E}-05 \\ 3.5 & 0.95 & 1.29 \mathrm{E}-3 & 2.83 \mathrm{E}-04\end{array}$




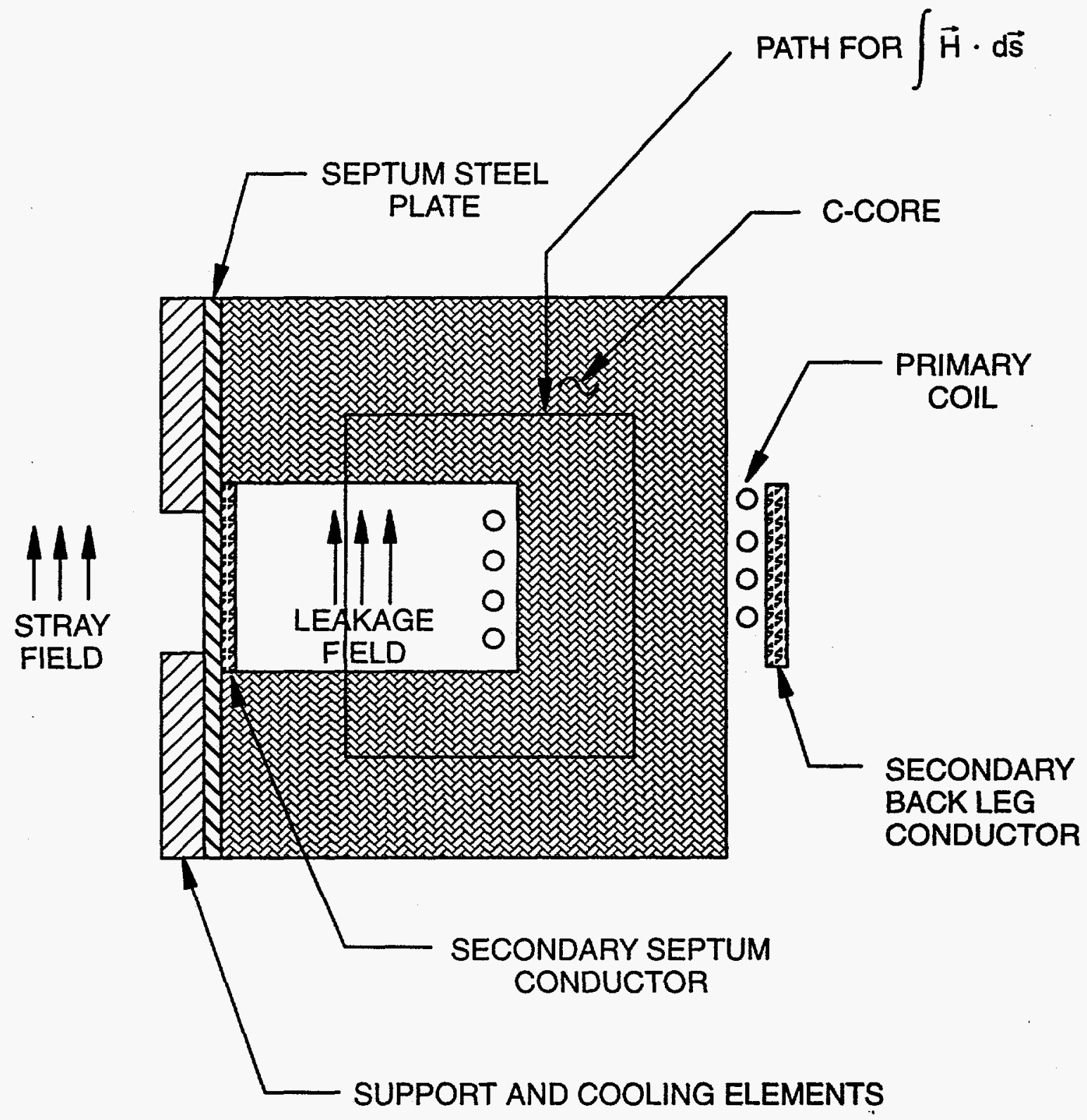

Figure 1. Schematic cross section of the eddy current septum magnet. 


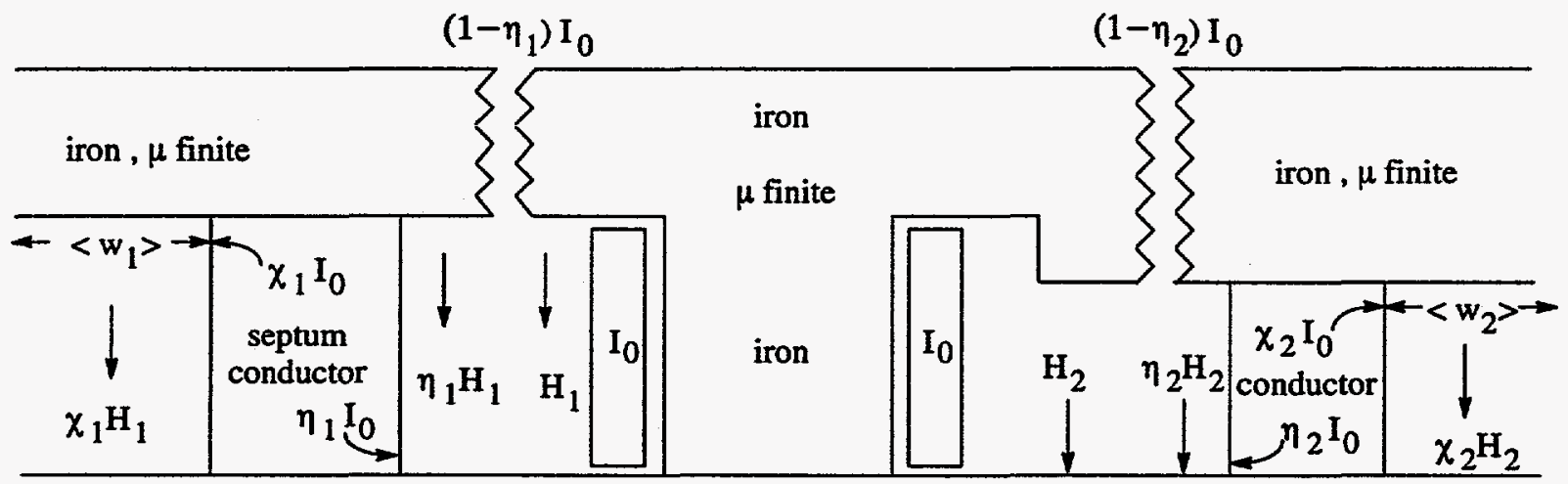

Figure 2. Model for global view. 


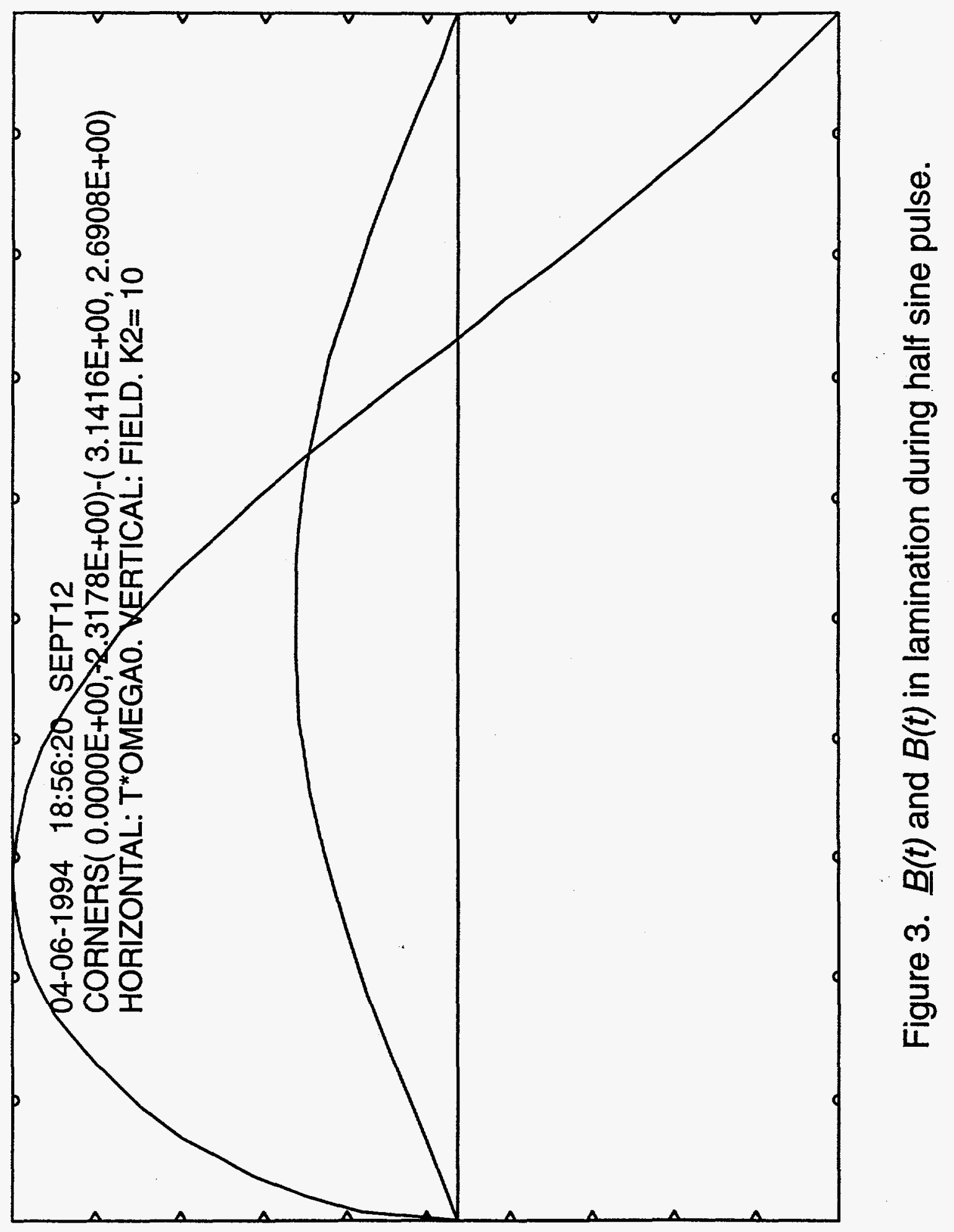




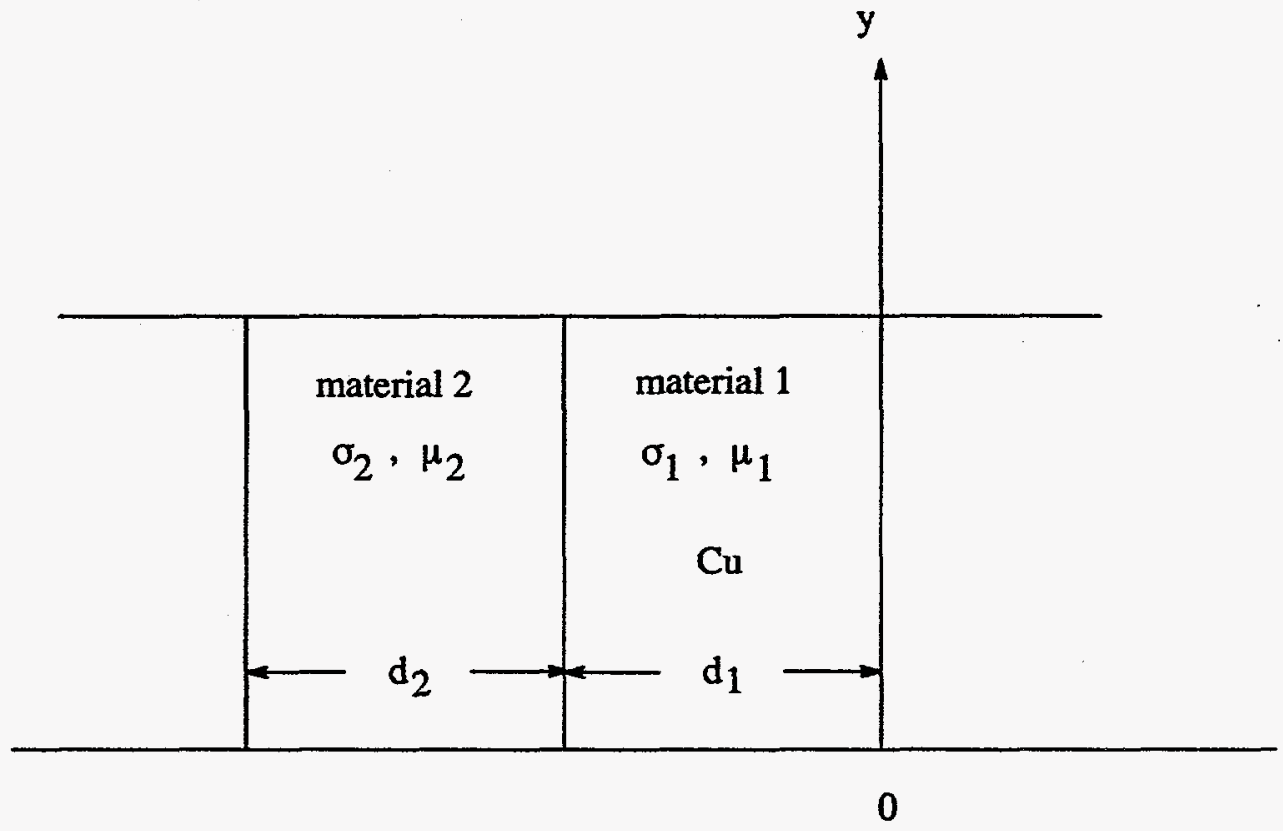

Figure 4. Schematic drawing of twocomponent septum. 


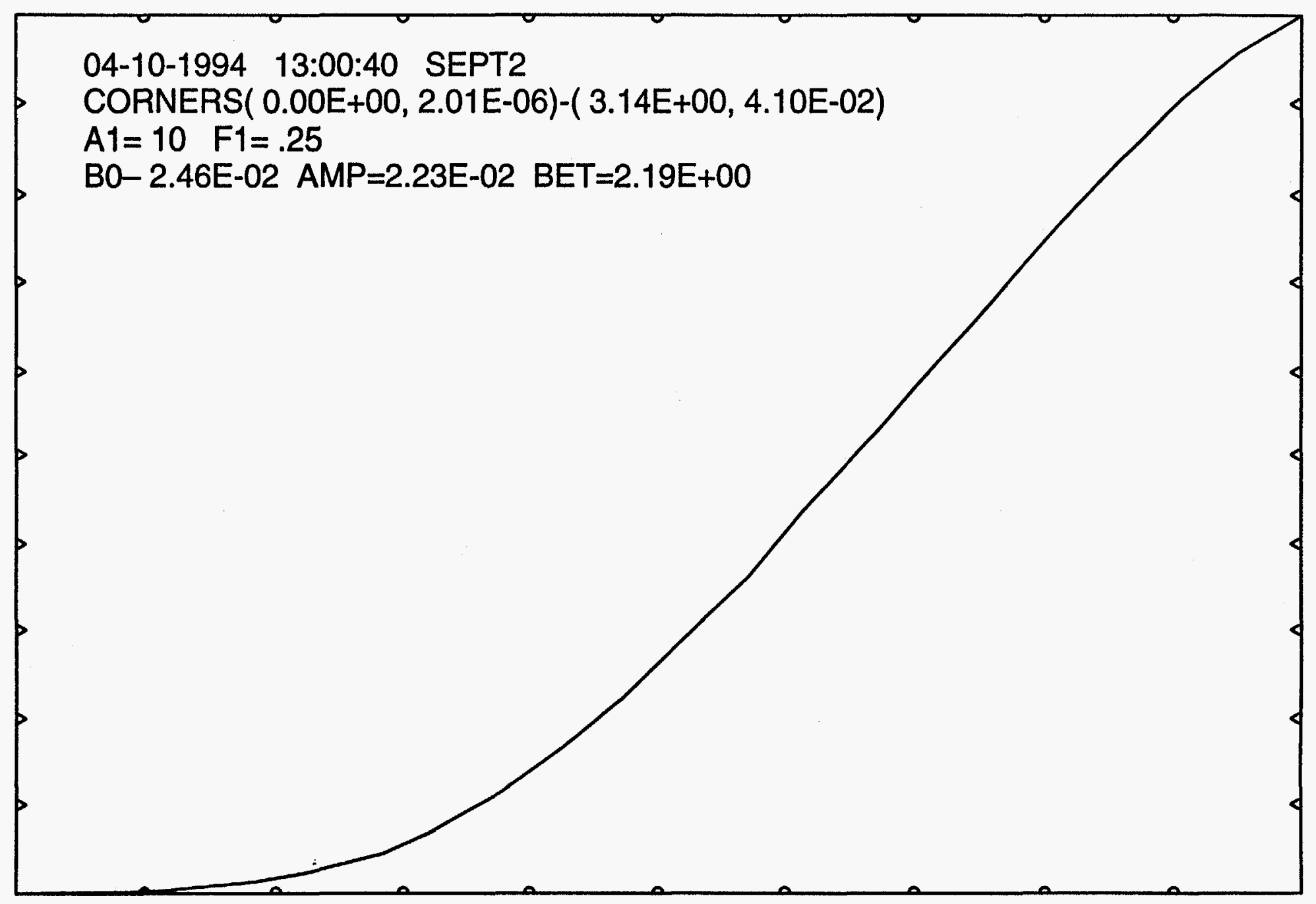

Figure $5 . \mathrm{H}_{3}$ during half sine pulse for pure $\mathrm{Cu}$ septum. 


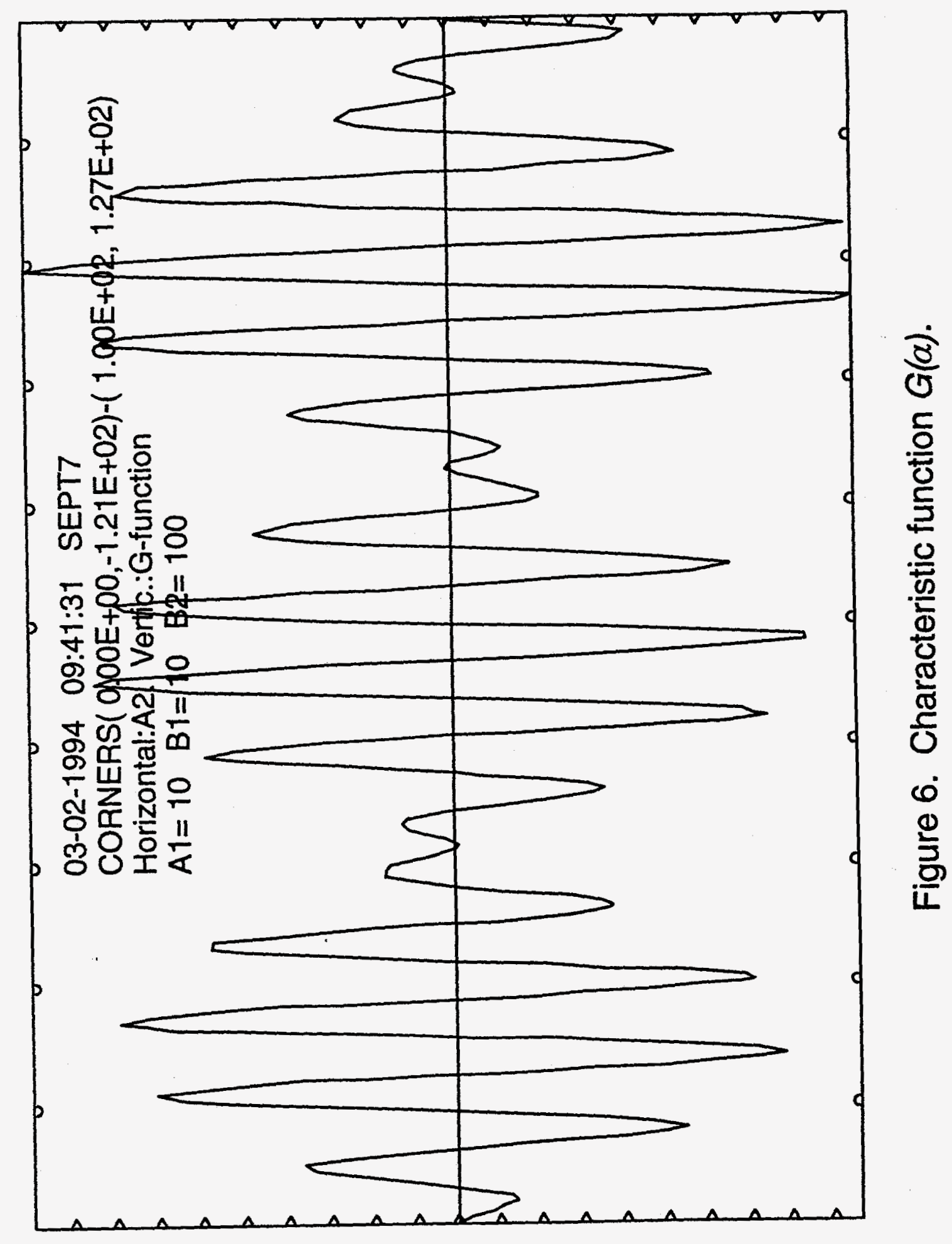




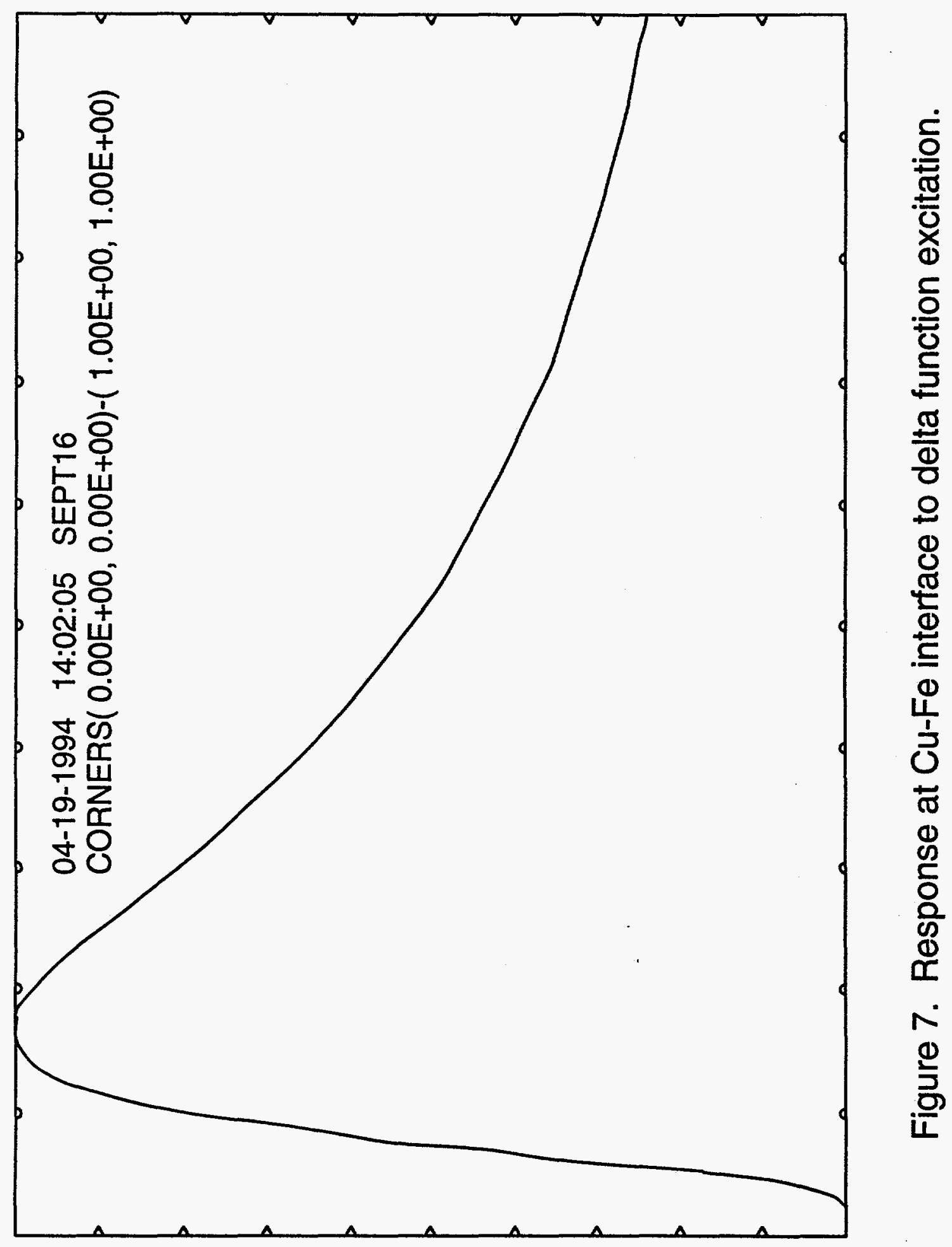




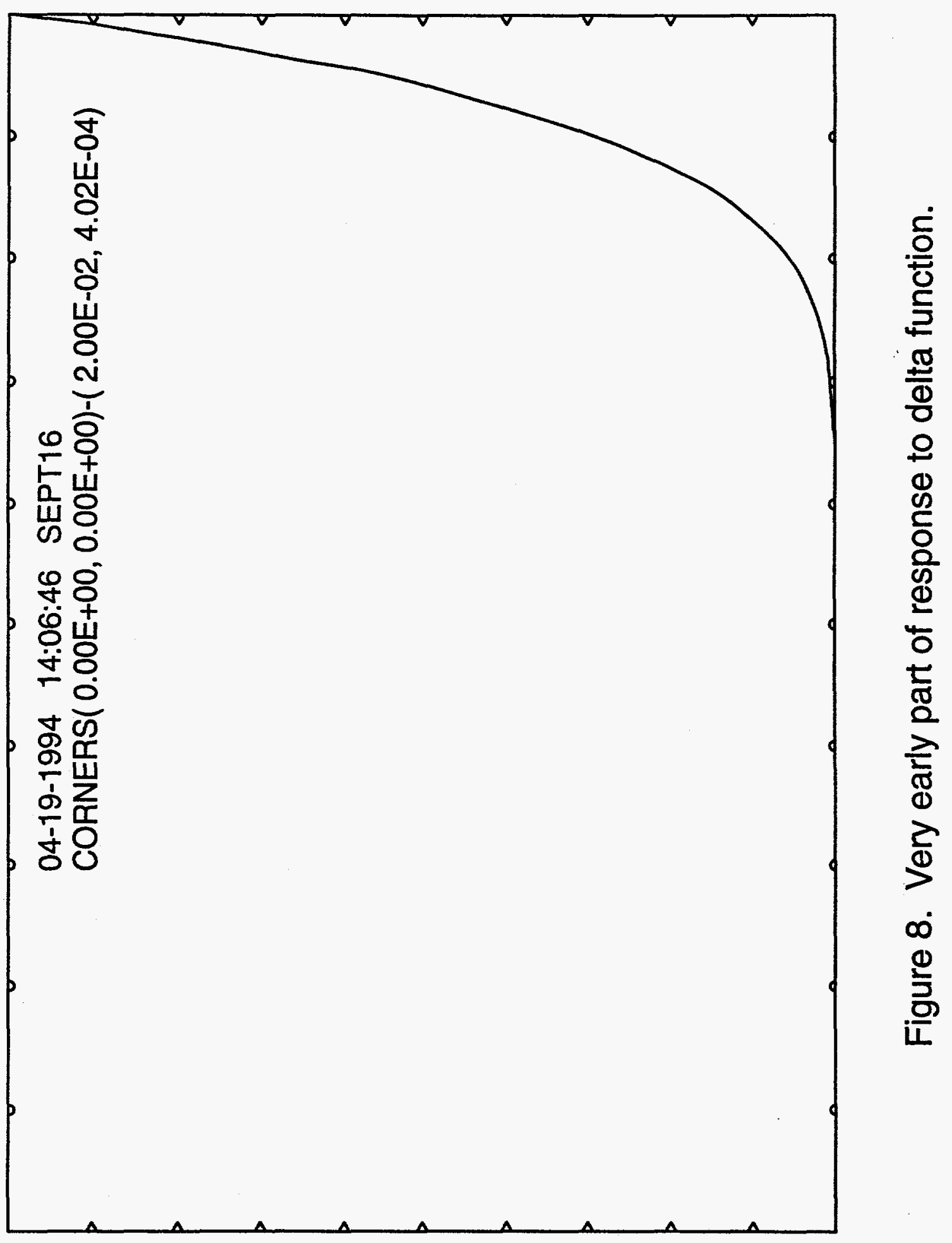




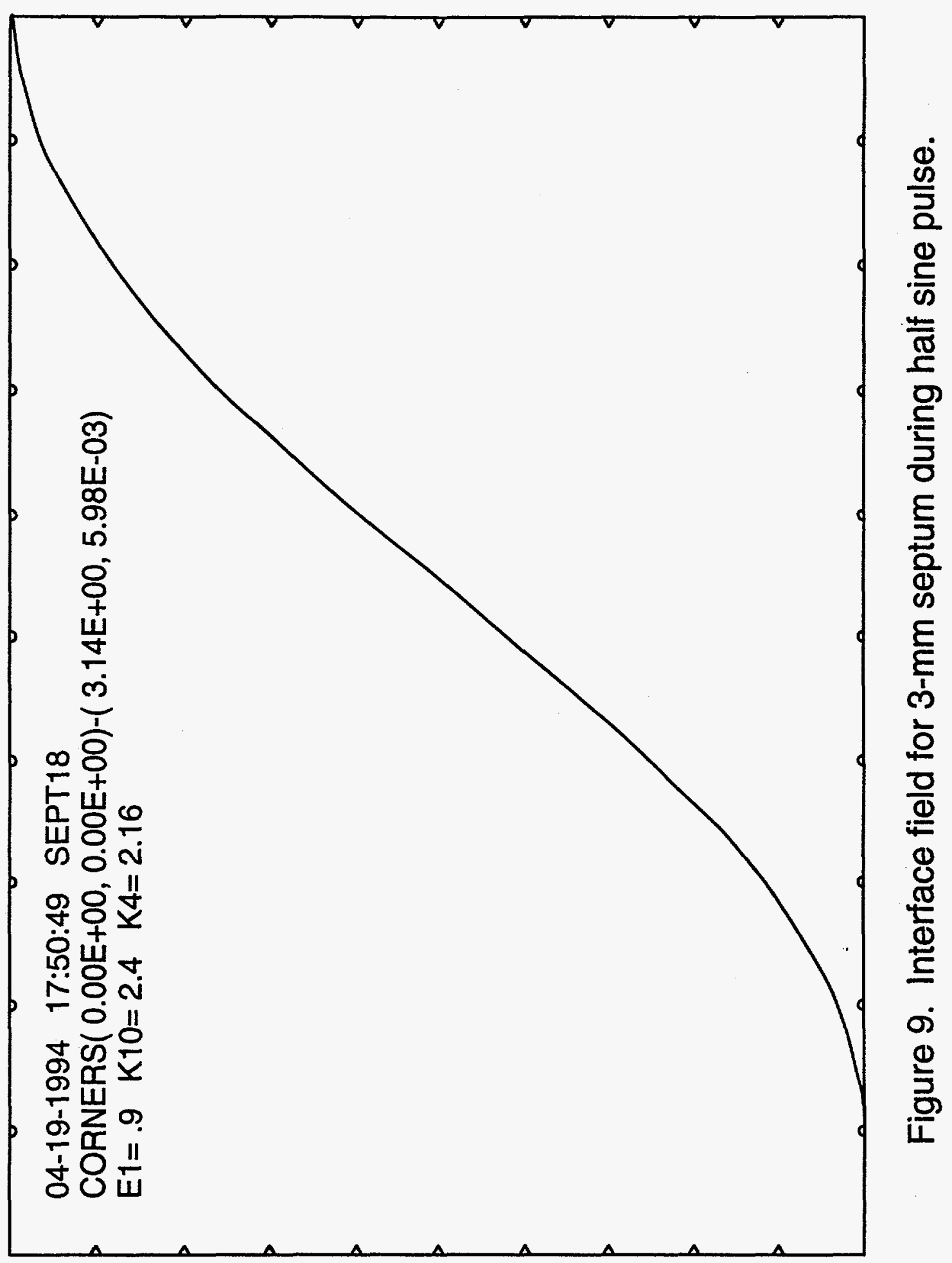




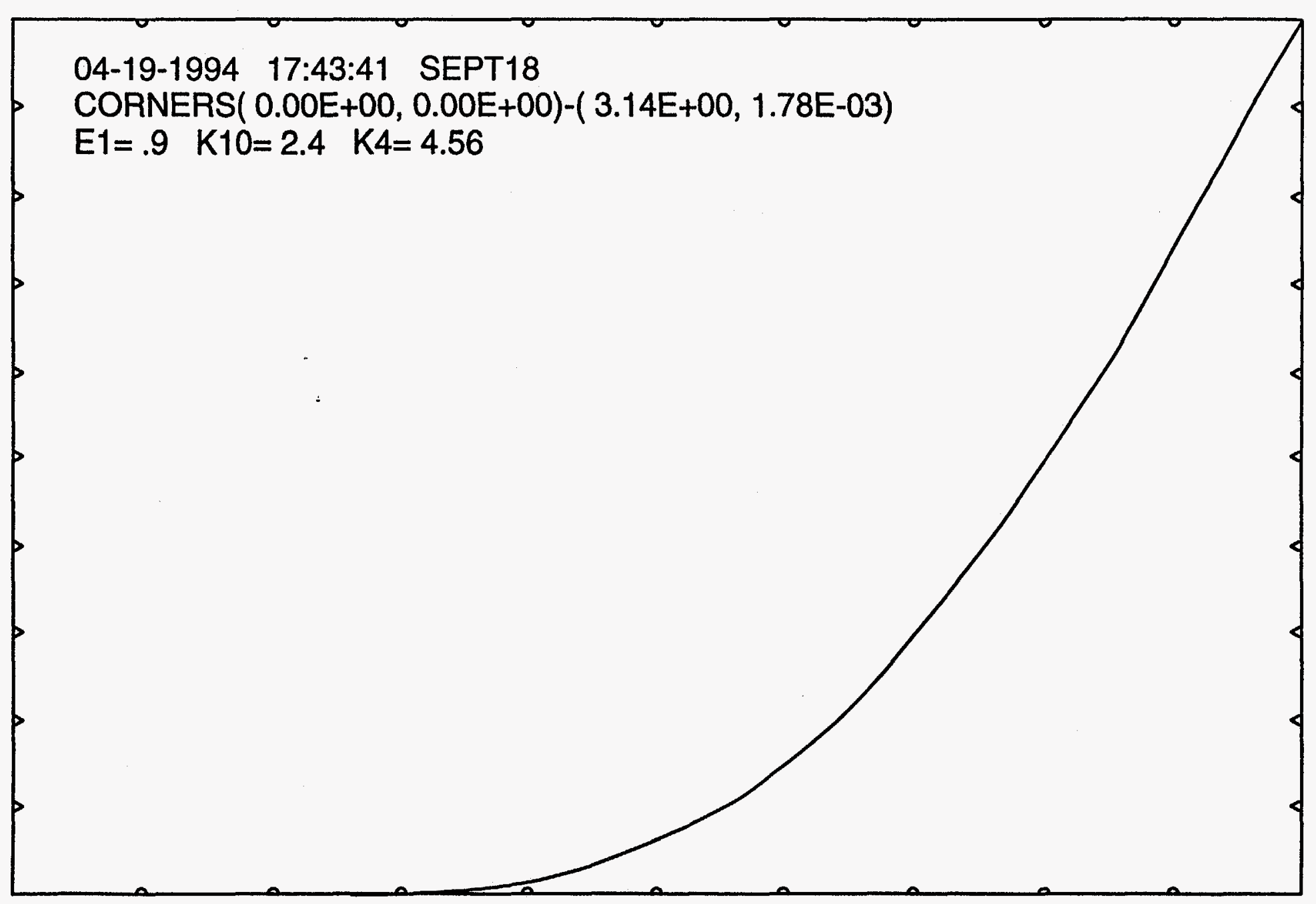

Figure 10. End field for 3-mm septum during half sine pulse. 


\title{
Septum Reset Pulse Design
}

\author{
Klaus Halbach \\ Argonne National Laboratory and US Lawrence Berkeley Laboratory
}

I assume a periodic set of forward use pulses and reset pulses and do the analysis after a stationary state has been reached. Time is measured in units of the longest time constant associated with the $\mathrm{Cu}-\mathrm{Fe}$ septum, given in equ.(12.3) of my septum report. Figure 1 shows schematically the sequence of events, with all fields drawn with positive values (to avoid sign errors), knowing that some field values will have to be negative. The field values indicated are the $\mathrm{H}$ fields in the septum iron. At $t=0, H=H_{0}$, which is "left over" from the reset pulse. The forward pulse starts and, on a time scale not visible on this graph, increases until the iron reaches the field $H_{s}$ where it begins to saturate, and I assume that it stays there for a while, discussed below. If the iron would not saturate, the field from the pulse would increase to the peak value $H_{0}+d H_{p}$, the forward pulse contributing $d H_{p}$. The important "use" part of that pulse, during which saturation has not caused any damage, is $H_{s}-H_{o}=d H_{u}$. That is the field that one has to make so large that the iron saturation does not occur before the driving pulse current has reached its maximum, and it can also be obtained with the help fo the septum report. After the primary current has stopped, the eddy currents in the iron will decay with a much smaller time constant than normal because of the low permeability associated with the saturated iron. After a short time (compared to the high permeability time constant) the permeability will go up again and the fields decay with the high permeability time constant. When the reset pulse is applied, at $t=t_{I}$, the field has decayed to $H_{s}$ $\exp \left(-t_{1}\right)$. After applying a reset pulse of amplitude $H_{r}$ and letting $t_{2}$ seconds elapse, the field should be again $=H_{0}$, giving

$$
H_{0}=\left(H_{s} e^{-t_{1}}+d H_{r}\right) e^{-t_{2}}=H_{s}-d H_{u} .
$$

This gives for the amplitude of the reset pulse

$$
d H_{r}=-e^{t_{2}}\left(d H_{u}-H_{s}\left(I-e^{-t_{3}}\right)\right)
$$

Clearly $d H_{r}$ increases both with $d H_{u}$ and $t_{2}$. There are, of course, some conditions that one should not violate. For instance, the largest useful amplitude for the reset pulse is given by the condition that the iron should not saturate in the reverse direction. While this analysis is very primitive, it gives at least some insight into what is possible and useful and what cannot be characterized that way. In particular it allows, combined with the information extractable from the septum report, the pain to be balanced between the time $t_{2}$ ( $t_{3}$ is fixed by the repetition rate) and the amplitude $d H_{r}$ of the reset pulse. 


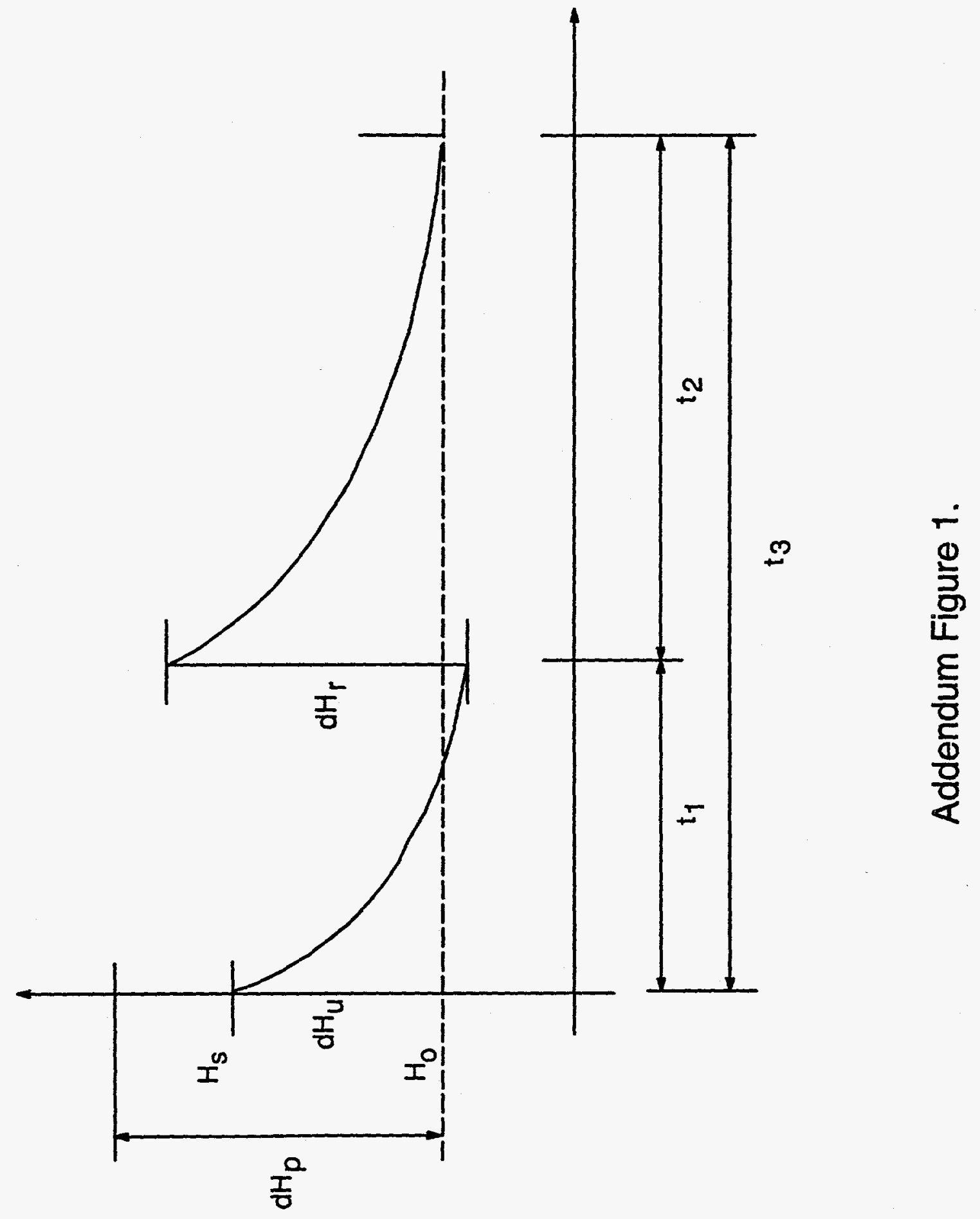

